\title{
Nuclear Effects on Bremsstrahlung Neutrino Rates of Astrophysical Interest
}

\author{
Sabin Stoica \\ Horia Hulubei National Institute of Physics and Nuclear \\ Engineering, P.O. Box MG-6, 76900 Bucharest-Magurele, Romania \\ J.E. Horvath \\ $I A G / U S P$ \\ Av. M. Stéfano 4200, Agua Funda, 04301-904 São Paulo SP, Brazil
}

November 4, 2018

\begin{abstract}
We calculate in this work the rates for the neutrino pair production by nucleon-nucleon bremsstrahlung taking into account the full contribution from a nuclear one-pion-exchange potential. It is shown that if the temperatures are low enough $(T \leq 20 \mathrm{MeV})$, the integration over the nuclear part can be done for the general case, ranging from the completely degenerate (D) to the non-degenerate (ND) regime. We find that the inclusion of the full nuclear contribution enhances the neutrino pair production by $n n$ and $p p$ bremsstrahlung by a factor of about two in both the D and ND limits when compared with previous calculations. This result may be relevant for the physical conditions of interest in the semitransparent regions near the neutrinosphere in type II supernovae, cooling of neutron stars and other astrophysical situations.
\end{abstract}

\section{Introduction}

Neutrino production processes in type II supernovae (SNII) and proto-neutron stars has received recently a considerable boost because of their importance in understanding of a 
variety of interesting phenomena like the very explosion mechanism, aspects related to the SNII nucleosynthesis, the interpretation of the neutrino signal from SN, the thermalization and cooling mechanisms, among others.

Several physical processes producing neutrinos have been investigated. The most important ones are electron scattering, electron-positron annihilation, pion-nucleon and NN scattering, NN bremsstrahlung, plasmon- and photo-neutrino production. Their relative importance for different stages of the early proto-neutron star evolution (including the explosion itself) is a consequence of quite different functional dependences on $\rho, T$. An analogous statement can be carried over to the great deal of work done on the cooling of mature neutron stars [1].

One of the important questions in supernova physics is the actual post-bounce evolution driven by neutrino emission, thought to be crucial for the long-term delayed shock revival driven by neutrinos (the Wilson mechanism). Neutrino production and thermalization were, for a long time, assumed to be dominated by pair annihilation and electron scattering respectively; at least for the $\nu_{\mu}$ and $\nu_{\tau}$ species (given that charged current reactions are certainly the main source for $\nu_{e}$ 's). Recenty, however, increasingly accurate calculations revealed that the energy transfer rate in neutrino $\left(\nu_{\mu}\right)$ - nucleon scattering may be up to one order of magnitude larger than the previous estimations, making this process competitive with the $\nu_{\mu}$-electron scattering as an equilibration mechanism [4], [6], [9]. Also, similar calculations for the total neutrino emissivity from NN bremsstrahlung indicate that this process may compete with $e^{+} e^{-}$annihilation [6], [10], [15] as a source for the $\nu_{\mu}$ and $\nu_{\tau}$ neutrinos. Bremsstrahlung rates have also been revisited for those nondegenerate to semidegenerate conditions, including the potentially important $n p$ channel. As a general feature of nucleon scattering, NN bremsstrahlung and URCA processes, it has proved quite difficult to treat the strong $\mathrm{NN}$ interaction matrix elements which are responsible for the $\nu$ production together with the weak interactions.

In the early calculations this interaction has been treated either by computing the overlap integrals associated with the initial and final nucleon wave functions [16] or through the use of a Fermi liquid parametrization [15]. Later on, a NN potential based on the one-pion-exchange (OPE) approximation has been deduced by several authors [14, [6], 
[5], [13], [7, [8]. However, in all these papers the full momentum transfer dependence of the OPE potential has been approximated (being, for instance replaced by the Fermi momentum) or even the whole square of the matrix element taken as a constant [8], [3]. However, for an accurate computation of these processes (and thus a realistic computation of the post-bounce evolution of type II supernovae) an accurate treatment of the nuclear potential is certainly required.

We present in this work a method for treating explicitly the momentum dependence of the OPE potential involved in the calculation of the neutrino pair production from NN bremsstrahlung. Actually, the method is rather general and could be also used for the treatment of other processes involving $\mathrm{NN}$ potential. We show that in particular physical conditions, characterized by temperatures $T<m_{\pi}^{2} / m \sim 20 \mathrm{MeV}$, the integral over the nuclear matrix element collapses, in a good approximation, to an integral which is independent of angles. The remaining part of the ME contribution, which depends only on the nucleon and neutrino energies, can be easily integrated numerically. Some results of this evaluation for non-degenerate and degenerate nucleons are presented.

\section{Calculations}

The total volumetric emissivity for the neutrino pair production by nucleon bremsstrahlung is given by

$$
Q_{\nu}=\frac{2 \pi}{\hbar} \int\left[\Pi_{1}^{4} \frac{d^{3} \mathbf{p}_{i}}{(2 \pi)^{3}}\right] \frac{d^{3} \mathbf{q}_{\nu}}{(2 \pi)^{3} 2 \omega_{1}} \frac{d^{3} \mathbf{q}_{\bar{\nu}}}{(2 \pi)^{3} 2 \omega_{2}} \omega\left(s \Sigma|M|^{2}\right)(2 \pi)^{3} \delta^{4}(P) F(f)
$$

where

$$
F(f)=f_{1} f_{2}\left(1-f_{3}\right)\left(1-f_{4}\right)
$$

is the product of Fermi functions for the initial $(1,2)$ and final $(3,4)$ nucleons $f_{i}=$ $\left(\exp ^{\frac{E_{i}-\mu_{i}}{T}}+1\right)^{-1}$. In eq.(1) $\mathbf{p}_{i}, i=1,4$ and $\mathbf{q}_{\nu}, \mathbf{q}_{\bar{\nu}}$ are the nucleon and neutrino momenta, respectively; $\omega=\omega_{1}+\omega_{2}$ are the neutrino energies ; $s$ is a symmetry factor taking into account the symmetry of identical particles $(s=1 / 4$ for $n n$ and $p p$, and $s=1$ for the $n p$ channel) and $E_{i}$ and $\mu_{i}$ are the energies and chemical potentials of the nucleons. 
In the non-relativistic limit $E_{i} \sim m+\frac{\mathbf{p}_{i}^{2}}{2 m}$ and one defines the chemical potential $\hat{\mu}=\mu-m$. With the introduction the non-dimensional quantities [8] $y=\frac{\hat{\mu}}{T} \quad ; u_{i}=\frac{\mathbf{p}_{i}^{2}}{2 m T}$ we write $f_{i}=\left(\exp ^{u_{i}-y_{i}}+1\right)^{-1}$. The degenerate (D) limit is achieved for $y>>1$, while in the non-degenerate (ND) limit $y<<1$.

In the OPE approximation the spin-summed NN matrix element (ME) for $n n$ and $p p$ interactions has been derived by several authors (see for instance [6]) and reads

$$
\begin{aligned}
s \Sigma_{\text {spins }}\left|M_{n n}\right|^{2}= & \\
=\frac{16}{4} \frac{64 G^{2} g_{A}^{2}}{\omega^{2}}\left(\frac{f}{m_{\pi}}\right)^{4} & {\left[\left(\frac{\mathbf{k}^{2}}{\mathbf{k}^{2}+m_{\pi}^{2}}\right)^{2}+\left(\frac{\mathbf{l}^{2}}{\mathbf{l}^{2}+m_{\pi}^{2}}\right)^{2}+\frac{\mathbf{k}^{2} \mathbf{l}^{2}}{\left(\mathbf{k}^{2}+m_{\pi}^{2}\right)\left(\mathbf{l}^{2}+m_{\pi}^{2}\right)}\right] \times } \\
& \times\left(\omega_{1} \omega_{2}-\mathbf{q}_{\nu} \mathbf{k} \mathbf{q}_{\bar{\nu}} \mathbf{k}\right) \equiv A \frac{\omega_{1} \omega_{2}}{\omega^{2}} M_{n n}
\end{aligned}
$$

where $g_{A}=1.26, f \simeq 1, A=64 G^{2} g_{A}^{2}\left(\frac{f}{m_{\pi}}\right)^{4} ; \mathbf{k}=\mathbf{p}_{1}-\mathbf{p}_{3}$ and $\mathbf{l}=\mathbf{p}_{1}-\mathbf{p}_{4}$ are the nucleon direct and exchange transfer momenta, respectively. In the non-relativistic approximation one can also neglect the neutrino momenta in eq.(3), since it is always small compared to the nucleon ones. For the sake of completeness we quote the corresponding ME for the $n p$ interaction [6]

$$
\begin{gathered}
s \Sigma_{\text {spins }}\left|M_{n n}\right|^{2}= \\
=\frac{1}{4} \frac{64 G^{2} g_{A}^{2}}{\omega^{2}}\left(\frac{f}{m_{\pi}}\right)^{4}\left[\left(\frac{\mathbf{k}^{2}}{\mathbf{k}^{2}+m_{\pi}^{2}}\right)^{2}+2\left(\frac{\mathbf{l}^{2}}{\mathbf{l}^{2}+m_{\pi}^{2}}\right)^{2}+2 \frac{\mathbf{k}^{2} \underline{1}^{2}}{\left(\mathbf{k}^{2}+m_{\pi}^{2}\right)\left(\mathbf{l}^{2}+m_{\pi}^{2}\right)}\right]\left(\omega_{1} \omega_{2}-\mathbf{q}_{\nu} \mathbf{k q}_{\bar{\nu}} \mathbf{k}\right) \times \\
\equiv A \frac{\omega_{1} \omega_{2}}{\omega^{2}}\left[2 M_{n n}-\left(\frac{\mathbf{k}^{2}}{\mathbf{k}^{2}+m_{\pi}^{2}}\right)^{2}\right]
\end{gathered}
$$

It is convenient to perform the integrals in the center-of-mass coordinate system and define the new variables introduced by Brinkmann and Turner [8]

$$
\begin{gathered}
\mathbf{p}_{+}=\frac{\mathbf{p}_{1}+\mathbf{p}_{2}}{2} ; \quad \mathbf{p}_{-}=\frac{\mathbf{p}_{1}-\mathbf{p}_{2}}{2} ; \quad \mathbf{p}_{3 c}=\mathbf{p}_{3}+\mathbf{p}_{+} ; \quad \mathbf{p}_{4 c}=\mathbf{p}_{4}+\mathbf{p}_{+} \\
\cos \gamma_{1}=\frac{\mathbf{p}_{+} \mathbf{p}_{-}}{\left|\mathbf{p}_{+}\right| \mid \mathbf{p}_{-}} ; \quad \cos \gamma_{c}=\frac{\mathbf{p}_{+} \mathbf{p}_{3 c}}{\left|\mathbf{p}_{+}\right| \mid \mathbf{p}_{3 c}} ; \quad \cos \gamma=\frac{\mathbf{p}_{-} \mathbf{p}_{3 c}}{\left|\mathbf{p}_{-}\right|\left|\mathbf{p}_{3 c}\right|}
\end{gathered}
$$


From the definition of the $u$ variables above, one can easily deduce the following relations

$$
u_{1,2}=u_{+}+u_{-} \pm 2\left(u_{+} u_{-}\right)^{1 / 2} ; u_{1,2}=u_{+}+u_{3 c} \pm 2\left(u_{+} u_{3 c}\right)^{1 / 2}
$$

We now address the $n n$ and $p p$ expresion of the OPE potential and calculate the corresponding emissivity. After some algebra one can express the matrix element eq.(3) in terms of the scalar combinations $a_{s}=\mathbf{k}^{2}+\mathbf{l}^{2}$ and $a_{p}=\mathbf{k}^{2} \mathbf{l}^{2}$ as

$$
M_{n n}=\left(3-m_{\pi}^{2} \cdot \frac{a_{s}\left(3 a_{p}+2 m_{\pi}^{2} a_{s}\right)+m_{\pi}^{2}\left(7 a_{p}+6 m_{\pi}^{2} a_{s}\right)+3 m_{\pi}^{6}}{a_{p}\left(a_{p}+2 m_{\pi}^{2} a_{s}\right)+m_{\pi}^{4}\left(a_{s}^{2}+2 a_{p}\right)+2 m_{\pi}^{6} a_{s}+m_{\pi}^{8}}\right) \equiv\left(3-M_{n n}^{c o r r}\right)
$$

Thus, the contribution of the nuclear ME can be splitted into a (already calculated) constant term and a correction to be evaluated. It is worth mentioning that the main part of the nuclear potential contribution (the constant term) is just 3, i.e. the limit to which the expression eq.(3) converges when the pion mass is neglected compared to the nucleon momentum transfer. The $a_{p}$ and $a_{s}$ terms have the explicit expressions $a_{s}=2 d(x+1 / 2 z) \quad ; \quad a_{p}=d^{2}\left[x(x+z) \sin ^{2} \gamma+1 / 4 z^{2}\right]$, with $d=2 m T, x=2 u_{3 c}$ and $z=\frac{\omega}{T}$. Again, after some algebra the correction part is cast in the form

$$
M_{n n}^{c o r r}=m_{\pi}^{2} \times \frac{A_{1}-A_{2}\left(c_{g}+s_{g} \cos \phi\right)^{2}}{B_{1}-B_{2}\left(c_{g}+s_{g} \cos \phi\right)^{2}+B_{3}\left(c_{g}+s_{g} \cos \phi\right)^{4}}
$$

where $c_{g}=\cos \gamma_{1} \cdot \cos \gamma_{c} ; s_{g}=\sin \gamma_{1} \cdot \sin \gamma_{c}$ and $\phi$ is the difference between the azithmutal angles corresponding to $\gamma_{1}$ and $\gamma_{c}$ (in spherical coordinates).

The coefficients $A_{i}$ and $B_{j}$ are polynomials of degree eight in the product $\left(m_{\pi}^{2} m T\right)$, depending only on variables $x$ and $z$ but otherwise independent of the angles. $B_{3}$ is also binomial having a coefficient proportional to $(2 m T)^{4}$, while $B_{1}$ and $B_{2}$ contain terms proportional to $m_{\pi}^{4}, m_{\pi}^{6}$ and $m_{\pi}^{8}$. Therefore, whenever $m T<m_{\pi}^{2}$ (or equivalent, $T<$ $20 \mathrm{MeV}), B_{3}$ can be neglected because its smallness compared to the other terms. This approximation can also be checked numerically. The results of integration over $M_{n n}^{\text {corr }}$ with and without the term $B_{3}$ differ each other by a very small amount, (less than $2 \%$ ) and greatly simplifies the calculation. The remaining integral over $\phi$ can be performed analytically yielding 


$$
I_{\phi}=2 \pi \frac{A_{2}}{B_{2}}=m_{\pi}^{2} \frac{3 d(2 x+z)+7 m_{\pi}^{2}}{2\left(d^{2}(x+z)+4 m^{2} T^{2} z^{2}+d m_{\pi}^{2}(2 x+z)+\frac{1}{4} d^{2} z^{2}+m_{\pi}^{4}\right)}
$$

The remaining integral over the other variables can be calculated by combining analytical and numerical techniques, for both the ND and D limits.

\section{Results}

We estimated first the total neutrino emissivity for the processes $n n \nu \bar{\nu}$ and $p p \nu \bar{\nu}$ since they involve the same contribution from the nuclear potential in both the ND and D cases. For comparison we normalize to the results of Thompson, Burrows and Horvath (2000) [3] valid for a constant matrix element and arbitrary degeneracies. Denoting their result for the $n n$ (or $p p$ ) neutrino emissivity with $Q_{b}^{n n}$ we find an underevaluation of their calculation of a factor of about two as compared with our result. More precisely we found

$$
\frac{Q_{\nu}^{n n}-Q_{b}^{n n}}{Q_{b}^{n n}}=1.065
$$

in the ND limit. Furthermore, this difference is independent of $y$ as long as the ND limit applies and $T<m_{\pi}^{2} / m$. Care should be taken in this comparison since instead of sticking strictly to the high-momentum limit (a procedure that would have required the presence of the "3" prefactor), Thompson, Burrows and Horvath (2000) have introduced a fudge factor $\xi$ to embrace all those effects. In terms of that quantity we obtain $\xi \sim 2$; or in other words, that the "true" bremsstrahlung emissivity is about $2 / 3$ of its highmomentum limit.

In the D limit, and adopting the same conditions of Flowers, Sutherland and Bond (1975) we obtain

$$
\frac{Q_{\nu}^{n n}-Q_{b}^{n n}}{Q_{b}^{n n}}=1.0578
$$

The neutrino emissivity for the case of the $n p \nu \bar{\nu}$ process can be calculated analogously using an analogous procedure to obtain $M_{n p}^{c o r r}$, but given that in general the chemical potentials of the neutrons and protons are different from each other this contribution is 
not so easily evaluated. We shall address the emissivity of this process and the general case for arbitrary degeneracy of all processes in a forthcoming paper.

\section{Conclusions}

In this work we developed a general method for calculating the total emissivity of the neutrino pair production from $\mathrm{NN}$ bremsstrahlung, taken into account the full contribution of an OPE nuclear potential. We have showed that for particular physical conditions, characterized by $T<20 \mathrm{MeV}$, the multiple integral appearing in the emissivity formulae can be performed. Both in the ND and D regimes we found that the inclusion of the nuclear potential contribution produces neutrino emissivities which are $\sim 2 / 3$ of their respective high-momentum limits. The method for including the full contribution of a nuclear OPE potential in the calculation of the neutrino emissivites from NN bresstrahlung is rather general and allows the computation of all $n n, p p$ and $n p$ processes in both ND and D limits and also it could be used to treat other proceeses of astrophysical interest, in which $\mathrm{NN}$ interaction is important.

Some key issues still remain to be clarified for a full evaluation of the bremsstrahlung neutrino emissivity, the most important perhaps is the interplay between the correlations in the dense medium ([5], [4]) and the momentum dependence of the nuclear potential. Other refinements (like the inclusion of multipion exchange) are not expected to be crucial, at least in the ND or mildly degenerate conditions corresponding to the semitransparent post-shock conditions in a SNII, although they may be relevant in other situations. Another issue is the corresponence with the recently claimed reduction extracted in a model-independent analysis by Hanhart, Phillips and Reddy [21]. We have found that the inclusion of the full OPE potential indeed reduces the emission rates in both extreme limits, although a detailed comparison with that work has not been attempted here.

\section{Acknowledgement}

S.Stoica wishes to acknowledge Fundação de Amparo à Pesquisa do Estado de São Paulo for financial support to visit the IAG/USP. J.E. Horvath wishes to acknowledge the 
CNPq Agency (Brazil) for partial financial support.

\section{References}

[1] See S. Tsuruta, Phys. Rep. 292, 1 (1998) for a review.

[2] D. Lamb and C. Pethick, Astrophys. J., 209, L77 (1976).

[3] T. A. Thompson, A. Burrows and J.E. Horvath, Phys. Rev. C 62, 035802 (2000).

[4] A. Burrows and R. Sawyer, Phys. Rev. C 58, 554 (1998).

[5] S. Hannestad and G. Raffelt, Phys. Rev. D 51, 6635 (1995).

[6] S. Hannestad and G. Raffelt, Astrophys. J. 507, 339 (1998).

[7] N. Iwamoto, Phys. Rev. Lett. 53, 1198 (1984).

[8] R. P. Brinkmann and M. S. Turner, Phys. Rev. D 38, 2338 (1988).

[9] G. Sigl, Phys. Rev. D 56, 3179 (1997).

[10] A. Burrows, T. Young, P. Pinto, R. Eastman and T.A.Thompson, Astrophys. J.539, 865 (2000).

[11] G. Raffelt and D. Seckel, Phys. Rev. Lett. 67, 2605 (1998).

[12] G. Raffelt and D. Seckel, Phys. Rev. D 52, 1780 (1995).

[13] G. Raffelt, D. Seckel and G. Sigl, Phys. Rev. D 54, 2784 (1996).

[14] B. L. Friman and O. V. Maxwell, Astrophys. J., 232, 541 (1979).

[15] E. G. Flowers, P. G. Sutherland and J. R. Bond, Phys. Rev. D, 12, 2 (1975).

[16] J. N. Bahcall and R. A. Wolf, Phys. Rev. B 140, 1452 (1965).

[17] A. Burrows and T. J. Mazurek, Astrophys. J. 259, 330 (1982). 
[18] A. Benvenuti et al., Phys. Rev. Lett. 32, 800 (1974).

[19] F. J. Hasert et al., Phys. Lett. B 46, 138 (1973).

[20] S.J. Barish et al., Phys. Rev. Lett. 33, 448 (1973).

[21] C. Hanhart, D.R. Phillips and S. Reddy, Phys. Lett. B 499, 9 (2001); see also A.E.L. Dieperink, E.N.E. van Dalen, A. Korchin and R. Timmermans, nucl-th/0012073 (2000). 